\title{
Erratum
}

Eclogae Geologicae Helvetiae Vol. 98 (2005) 113-122

\section{A new species of the genus Besania Brough 1939 from the Middle Triassic of Canton Grisons (Switzerland) with a discussion of the phylogenetic status of the taxon}

Annette Herzog \& Toni Bürgin

Following the International Code of Zoological Nomenclature (Article 31.1.2), the newly erected species name Besania schaufelbergeri has to be replaced by the correct name Besania schaufelbergerae.

The reason for doing this was the usage of an incorrect gender (masculine instead of feminine). 